
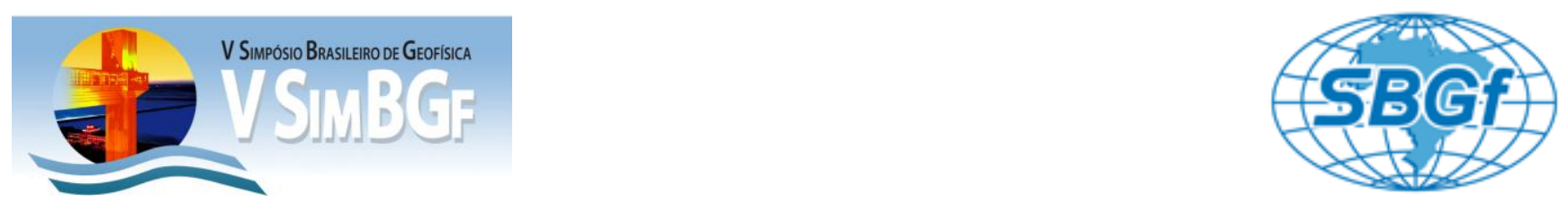

\title{
Segmentação de lâminas de rochas utilizando a morfologia matemática através da abordagem de Watershed
}

Sarah Soares Maduro de Oliveira

Carlos Eduardo Guerra

Universidade Federal do Oeste do Pará

Copyright 2012, SBGf - Sociedade Brasileira de Geofísica

Este texto foi preparado para a apresentação no V Simpósio Brasileiro de Geofísica, Salvador, 27 a 29 de novembro de 2012. Seu conteúdo foi revisado pelo Comitê Técnico do V SimBGt, mas não necessariamente representa a opinião da SBGf ou de seus associados. É proibida a reprodução total ou parcial deste material para propósitos comerciais sem prévia autorização da SBGt.

\section{Resumo}

Este trabalho apresenta uma aplicação da morfologia matemática na segmentação de lâminas de rocha, utilizando a abordagem de watershed para separar os grãos em contato. Primeiro, a metodologia foi testada em imagens sintéticas geradas através do sistema SpheroPolyhedra ${ }^{\mathrm{TM}}$, obtendo excelentes resultados. O próximo passo foi aplicar a metodologia em imagens de lâminas digitais extraídas de uma rocha arenito. A segmentação foi alcançada com alguns ajustes manuais.

\section{Abstract}

This paper presents an application of mathematical morphology in the segmentation of petrographic slides, using a watershed approach to separate the grains in contact. First, the methodology was tested on synthetic images generated by the SpheroPolyhedra TM system, obtaining excellent results. The next step was to apply the methodology in digital images of slides taken from a sandstone rock. The segmentation was achieved with some manual adjustments.

\section{Introdução}

O processamento de imagens de lâminas de rocha é importante para diversos estudos geológicos, buscando dados como o tamanho e forma dos grãos, agregação, compactação, dentre outros, para a determinação de fatores em pesquisas científicas e exploração no que se refere a manejo de solos, mineralogia, geologia, petrofísica e caracterização de reservatórios.

Como cita Banon \& Barrera (1988), é preciso estudar a geometria da rede de canais para caracterizar a permeabilidade da rocha e assim definir a energia necessária para retirar óleo, por exemplo.

\section{Morfologia Matemática - Teoria Básica:}

Uma imagem em níveis de cinza pode ser vista como uma matriz de pontos, denominados por pixels, e cada pixel, localizado na i-ésima linha e j-ésima coluna desta matriz, recebe um valor (geralmente um número inteiro não negativo) da escala de níveis de cinza. Para imagens binárias, os pixels são atribuídos a dois valores, 0 ou 1 .

A morfologia Matemática foi criada na década de sessenta e o desenvolvimento dos conceitos básicos, com uma sistemática prévia da teoria, foi apresentada na monografia de G. Matheron em 1975 e J. Serra em 1982.

Ela possibilita estudar as imagens com relação a mudanças na forma, tamanho e contorno, e é fundamentada em dois operadores básicos: erosão e dilatação.

A dilatação é a união das translações de um elemento estruturante (uma imagem com tamanho e forma conhecida), com cada pixel da imagem.

$$
A \oplus B=\left\{x \mid(B)_{X} \cup A\right\}
$$

Já a erosão é a interseção dessas translações, ou seja:

$$
A \Theta B=\left\{x \mid(B)_{x} \cap A\right\}
$$

A dilatação "engorda" a imagem, fecha buracos e conecta elementos, enquanto que a erosão aumenta o espaçamento entre objetos, diminui as áreas. Através das combinações destas operações, usando o mesmo elemento estruturante, pode-se criar diversos outros operadores, como abertura (uma erosão seguida por uma dilatação) e fechamento (uma dilatação seguida de uma erosão).

Outro operador frequentemente utilizado é o Gradiente Morfológico, definido, segundo Soille (1999), como a diferença aritmética entre a dilatação e erosão de uma imagem.

$$
\nabla_{b_{e}, b_{i}}(A)=\left(A \cup b_{e}\right)-\left(A \cap b_{i}\right)
$$

O operador gradiente é normalmente empregado em processos de segmentação. A segmentação visa dividir a imagem em unidades significativas, que apresentam algum tipo de similaridade, segundo Marques E Vieira (1999). Dentre as inúmeras técnicas disponíveis, destacamos a técnica de watershed, que utiliza a transformada de distâncias para ler a imagem como "bacias de drenagem". Os pixels mais distantes do background, i.é, com os valores mais altos na transformada de distâncias, são visto como os pontos mais fundos das "bacias" e, a partir deles, o programa passa a "inundar" a imagem. Onde os reservatórios de água se encontram são traçadas as linhas de watershed.

\section{Metodologia}

A metodologia proposta consiste em aplicar cinco etapas de processamento sobre a imagem alvo. Na primeira 
etapa, a imagem é lida e simplificada de forma a se eliminar ruídos e destacar os objetos para a segmentação. Se a imagem for multispectral, pode-se escolher uma banda ou uma combinação de duas ou mais bandas. Operadores de limiarização, aberturas e fechamento podem ser aplicados à imagem para a remoção de objetos e ruídos indesejados. A limiaraização de uma Imagem $A$,em níveis de cinza, gera uma imagem binária $B$, simplificada, onde $L_{i}$ e $L_{s}$ são os limiares inferior e superior respectivamente, ou seja, os pixels de A fora do intervalo limiar são colocados como zero em B e os pertencentes ao intervalos são colocados como um.

$$
\left\{\begin{array}{l}
B_{i j}=1, \text { se } A_{i j} \in\left[L_{i} . . L_{s}\right] . \\
B_{i j}=0, \text { caso contrário. }
\end{array}\right.
$$

$\mathrm{Na}$ Figura 1.A, tem-se uma imagem binária (já limiarizada) com a presença de ruídos. Na Figura 1.B temos a imagem resultado simplificada.

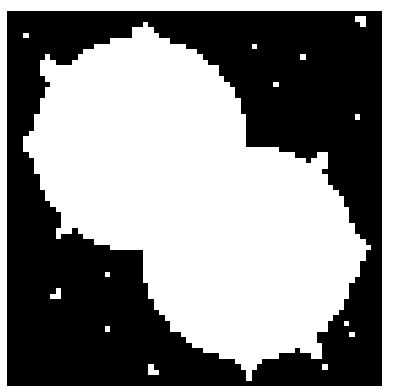

(A)

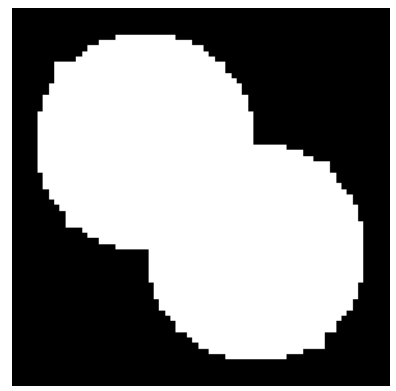

(B)
Figura 1 - Imagem binária com ruído $(A)$, imagem após a aplicação do operador de abertura (B).

$\mathrm{Na}$ segunda etapa, aplica-se a transformada de distâncias sobre a imagem simplificada para se obter marcações do background (fundo da imagem) e foreground (objetos relevantes). Como marcação de fundo, utilizou-se o inverso da transformada de distância e para marcação de objeto, a imagem foi erodida até o seu ponto mínimo (ponto verde na Figura 2.B).

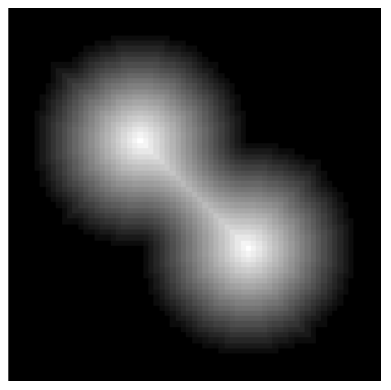

(A)

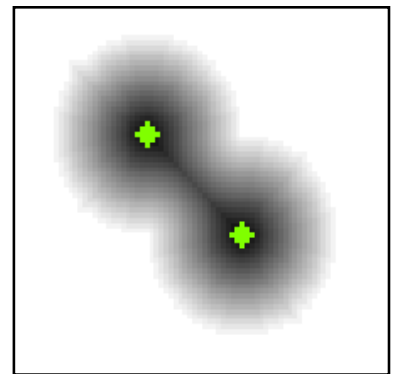

(B)
Figura 2 - Transformada de distância (A), inverso da transformada da distância (B), pontos verdes são os marcadores que foram utilizados.
$\mathrm{Na}$ terceira etapa, as marcações obtidas são empregadas no operador de watershed para obtermos a segmentação da imagem (Figura 3.A). Na quarta etapa, os contornos dos objetos são obtidos, aplicando o gradiente morfológico sobre a interseção entre o inverso da imagem segmentada (Figura 3.B) e a imagem simplificada (Figura 1.B). Uma quinta etapa pode ser necessária de forma a se remover ou atenuar excessos de linhas de segmentação, que podem separar erroneamente as partículas.

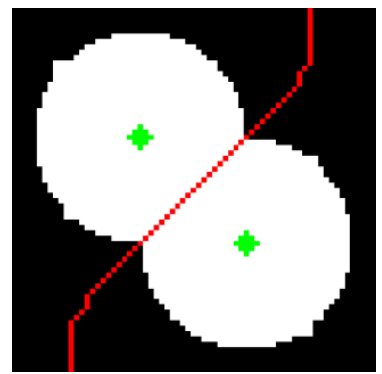

(A)

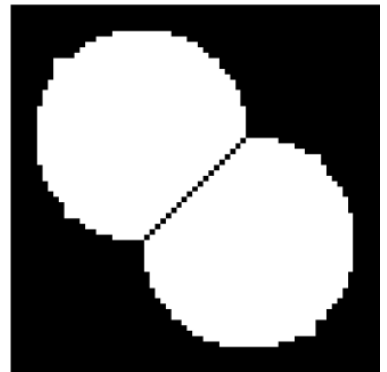

(B)
Figura 3 - Em (A), a segmentação de watershed (em vermelho) na imagem limiarizada e os marcadores em verde. Interseção entre o inverso da segmentação de watershed e a imagem limiarizada $(B)$.

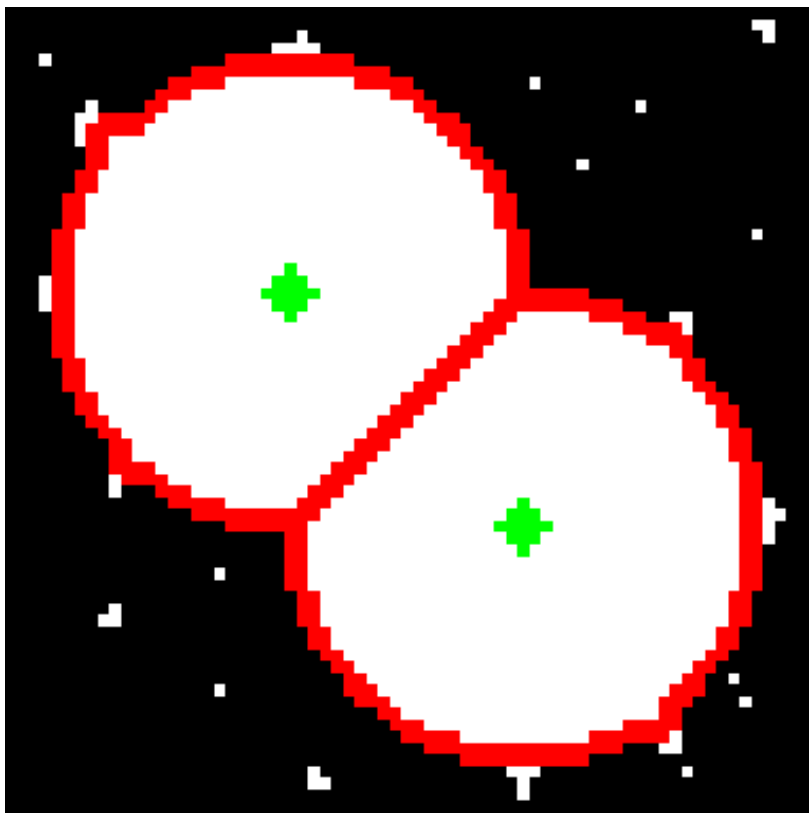

Figura 4 - Imagem original com as bordas traçadas pelo gradiente da imagem da Figura 3 (B).

Para o caso de imagens mais complexas, um programa interativo pode remover marcadores inesperados, onde o marcador clicado é subtraído da imagem, e assim uma segmentação diferente é traçada. 


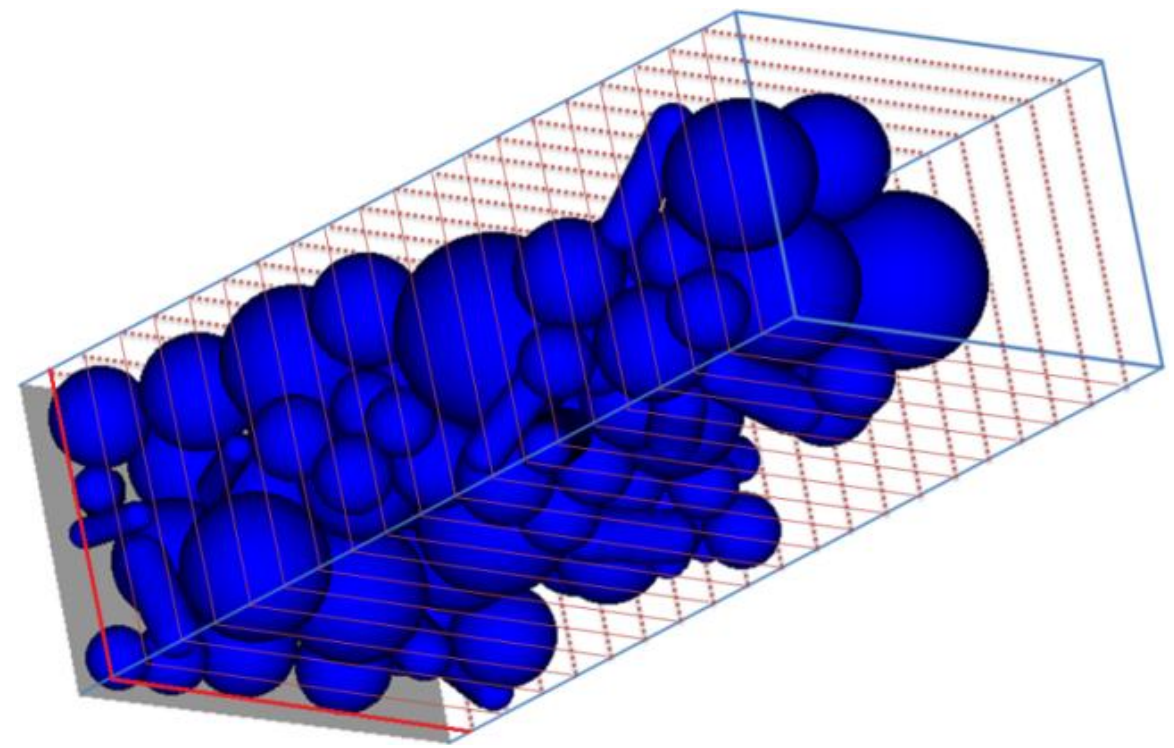

Figura 5 - Modelo tridimensional de uma lâmina de rocha. As linhas em laranja representam os níveis que foram cortados. Destacado em vermelho, está o corte que foi utilizado como exemplo de modelo de lâmina.

\section{Resultados}

Primeiramente utilizou-se o sistema SpheroPolyhedra ${ }^{\mathrm{TM}}$ para gerar um modelo tridimensional de formação, baseado no empacotamento de esferas e bastonetes em um invólucro retangular (Figura 5 ). O pacote gerado simula uma amostra da rocha. A seguir, foram feitos cortes transversais no modelo, com espaçamentos regulares, gerando lâminas individuais e armazenadas em forma de imagens.

Na Figura 6, tem-se a lâmina escolhida (destacada em vermelho na Figura 5) para o processamento. Seguindo a metodologia proposta, separou-se a imagem (RGB) da lâmina em três bandas e escolheu-se a banda R. No modelo adotado, a imagem da lâmina já está na forma binária, uma vez que o material das esferas, bastonetes e de preenchimento do invólucro são homogêneos. Ainda na mesma figura, nota-se que muitos grãos estão em contato entre si.

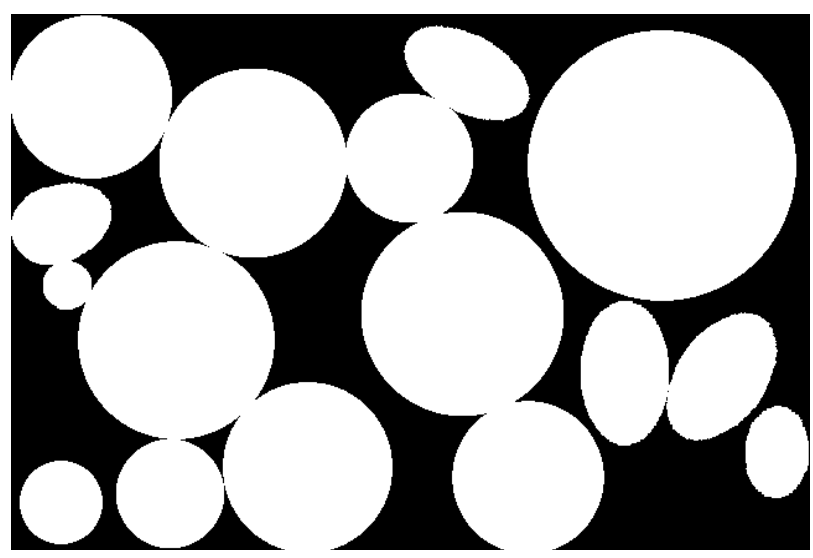

Figura 6 - Imagem binária do modelo.

Para estudos morfológicos e granulométricos, faz-se necessário a separação total dos grãos, o que pode ser feita através da aplicação de operadores morfológicos, rotulação de objetos e da transformada de watershed.A seguir, uma transformada de distância foi aplicada à imagem resultante, e os marcadores do background e foreground foram obtidos através do inverso e do ponto de mínimo da transformada de distância. A segmentação de watershed e o gradiente traçaram as linhas divisórias entre os objetos e os contornos.

A Figura 7 mostra o resultado do gradiente na interseção entre a marcação de watershed e a imagem original de modelo de lâmina de rocha criada no sistema SpheroPolyhedra ${ }^{\mathrm{TM}}$, onde as linhas em vermelho são os contornos traçados e os objetos em verde são as marcações utilizadas para diferenciar os objetos do background; a imagem resultante está em binário.

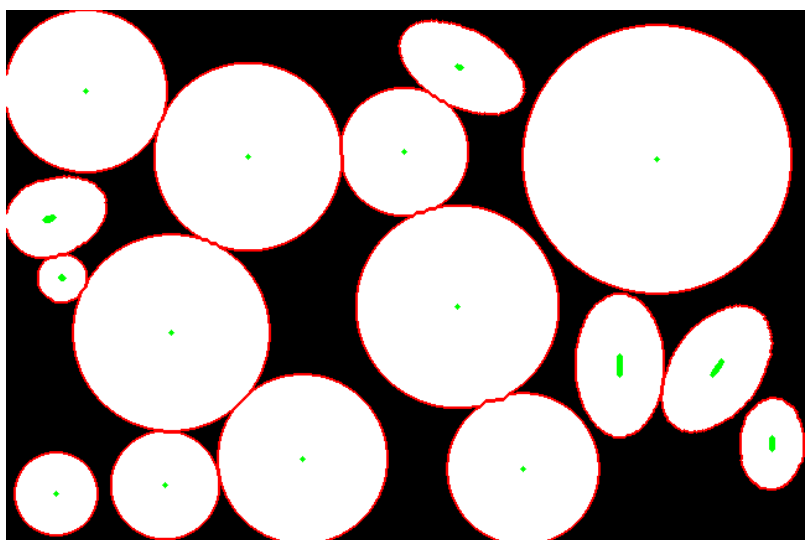

Figura 7 - Imagem em binário com as bordas (em vermelho) delimitadas pela segmentação de watershed e marcadores dos objetos em verde.

A seguir, a metodologia foi aplicada em uma imagem de lâmina de uma rocha arenito, (Figura 8), com dois líquidos de preenchimento (água e hidrocarboneto), e os mesmos passos foram seguidos, obtendo-se a imagem 
da Figura 9. Nota-se que a segmentação de watershed resultou em uma imagem super-segmentada com um excesso de rótulos, o que causou o particionamento errôneo dos grãos. Neste caso, utilizou-se uma remoção parcial dos marcadores de grãos (foreground) de forma a eliminar as marcações erradas. A imagem resultante foi novamente segmentada utilizando-se as marcações não subtraídas da imagem.

Percebe-se na imagem final (Figura 10), que a maioria dos objetos foi delimitada, exceto algumas partes mais escuras dos grãos que não receberam marcações e foram então vistas como parte do background, no caso, o líquido de preenchimento.

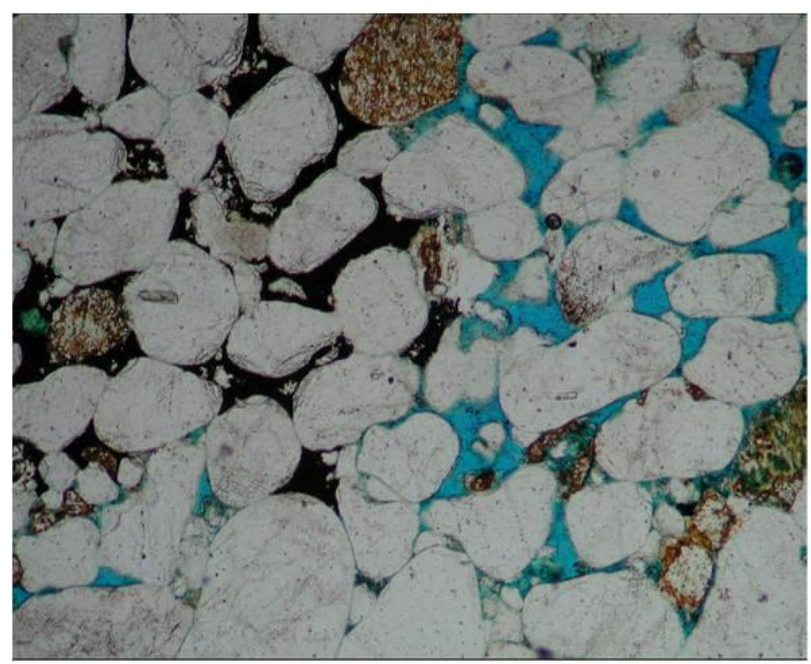

Figura 8 - Imagem de lâmina de arenito

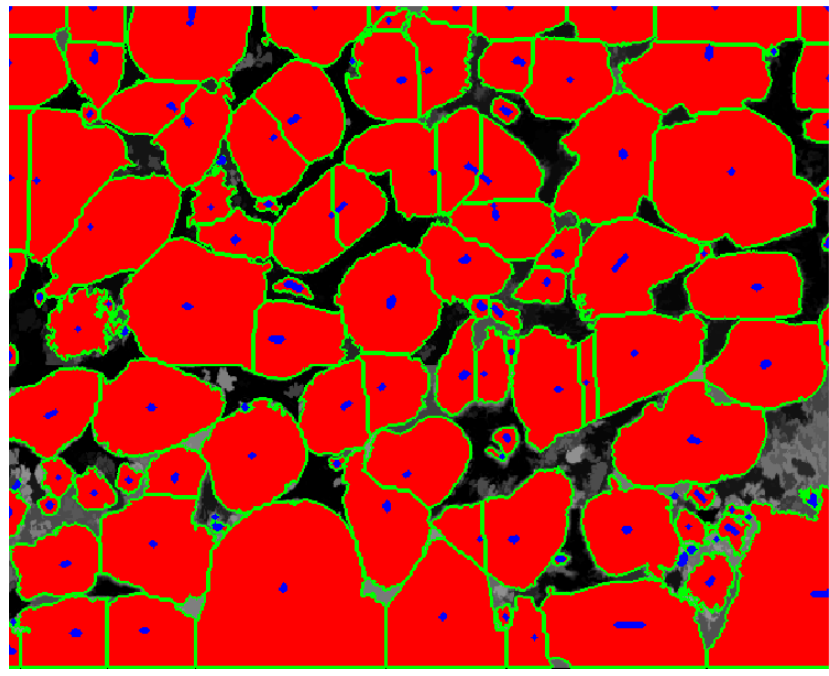

Figura 9-Imagem antes do ajuste manual.

Na Figura 11 tem-se a imagem da lamina segmentada, mostrando os contornos das partículas (bordas verdes sobre objetos vermelhos), os marcadores de foregrround e a separação das fases fluidas (amarelo para água e rosa para hidrocarbonetos).

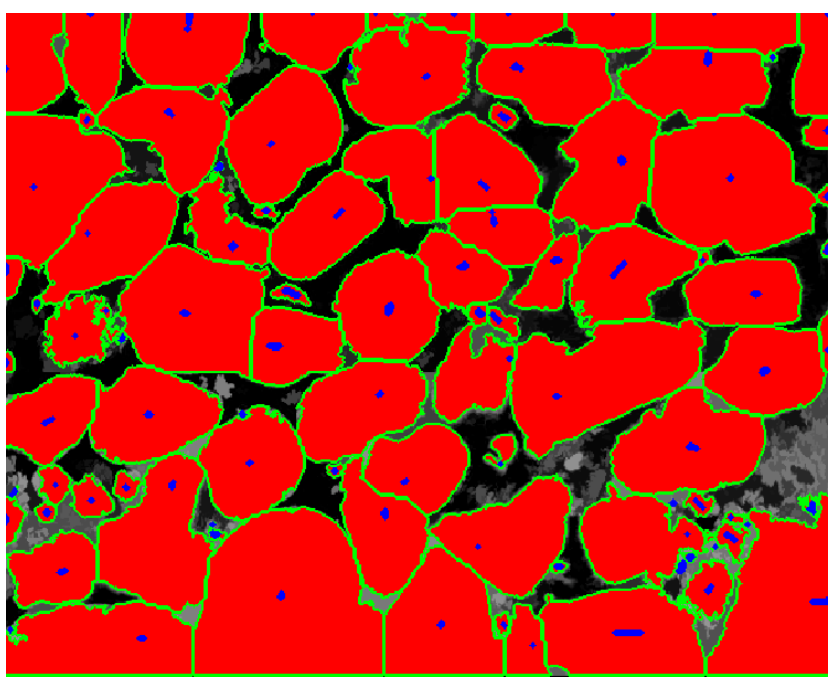

Figura 10 - Imagem após o ajuste manual.

Para a separação das fases fluidas, foi necessário aplicar a metodologia com duas limiarizações distintas, uma para separar a fase hidrocarboneto (fundo negro da Figura 8) e outra para separar a fase água (fundo azul da Figura 8).

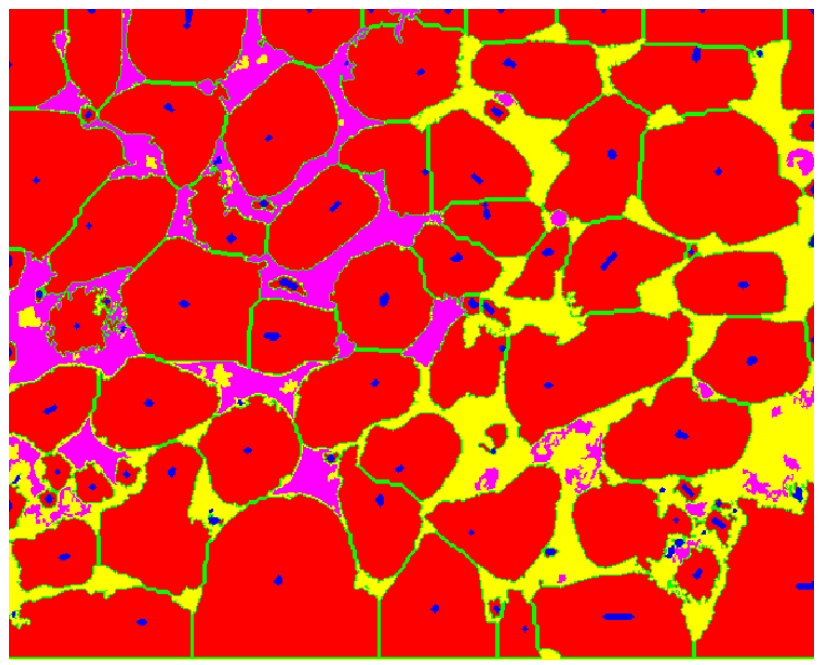

Figura 11 - Separação de todas as fases da imagem. Em magenta é caracterizado o hidrocarboneto, em amarelo, a água, em vermelho, os grãos (objetos que foram segmentados), os pontos em azul são as marcações do foregrouns, e em verde, o gradiente da imagem, as bordas.

\section{Discussão e Conclusões}

A metodologia aplicada nas imagens geradas pelo programa S3D SpheroPolyhedra ${ }^{\mathrm{TM}}$ se mostrou muito eficiente conseguindo um resultado satisfatório, segmentando os objetos com precisão (figura 7). Esse resultado era esperado, visto que no modelo utilizado, os 
objetos possuíam formas bem definidas (esferas ou bastonetes), o que facilita a segmentação de watershed, já que pela continuidade das formas, elas são mais facilmente erodidas até um único ponto (o ponto de marcação), evitando a supersegmentação.

Este problema foi visto claramente quando a metodologia foi aplicada a imagem de lâmina de rocha de arenito, já que os grãos na imagem possuem algumas descontinuidades quanto à forma e coloração, produzindo assim uma imagem binária que não é erodida facilmente até um único ponto de marcação.

O ajuste manual feito na quinta etapa, ainda mostrou alguns erros, que podem ser percebidos na imagem final (figura 10), como alguns grãos que não foram delimitados, pois não possuíam pontos de marcação (mesmo antes da retirada dos pontos em excesso), e marcações desnecessários que precisaram ser mantidas (todas as que tocavam as linhas de watershed). Finalmente, a metodologia proposta é o ponto de partida para um estudo mais completo de lâminas petrográficas, onde a segmentação de partículas e separação do background e das fases fluidas poderão ser empregados para o cálculo de porosidade, saturação e também a construção de modelos $3 \mathrm{~d}$ de meios porosos.

\section{Agradecimentos}

Agradeço a UFOPA, ao CNPq, e ao Laboratório de Sensoriamento Remoto e Geofísica pelas condições proporcionadas para a realização deste trabalho.

\section{Referências}

Woods, G \& E, Digital Image Processing Using Matlab; segunda edição. Prentice-Hall, Inc. 2002. 793p.

Banon, G. J. F. e J. Barrera. Bases da Morfologia Matemática para Análise de Imagens Binárias; segunda edição. INPE, São José dos Campos 1988.

Facon, J. Morfologia Matemática, Teoria e Exemplos. Editora Universitária Champagnat, Pontifícia Universidade Católica do Paraná, Curitiba, 1

Guerra, C.E, Ruela, A.X. Andrade. A.J.N. Watershed threshold and gray level morfology applied to object detecting in remote sensing and petrographics images. XII CSBGF, 2011.

Matheron. G, Serra. J. "The Birth of Mathematical Morphology". École Nationale Supérieure des Mines de Paris. 2000

Marques F, O; Vieira N, H. Processamento Digital de Imagens, Rio de Janeiro: Brasport, 1999. ISBN 8574520098.

Dmitry B. S, Guodong J, Tad W. P. Robust Determination of the Pore Space Morphology in Sedimentary Rocks. SPE Annual Technical Conference and Exhibition held in Denver, Colorado, U.S.A., 58 October 2003.
Vincent, L. Dougherty, E R. Morphological Segmentation for Textures and Particles. Digital Image Processing Methods, E. Dougherty. New York, 1994, Pages 43--102.

Smart Imaging Technologies, AlgART Laboratory, 3D Packing of Sphere-Polyhedra 2.2, http://smartimtech.com. 